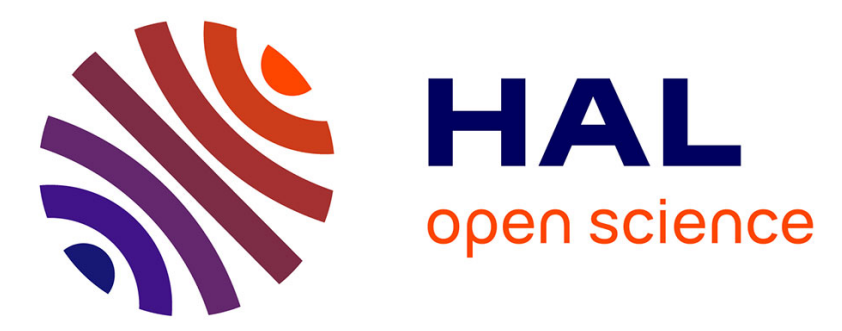

\title{
Exciton resonance reflectivity study of quantum well wires
}

\author{
V. Kochereshko, E. Ivchenko, A.V. Kavokin, P. Kopev, N. Ledentsov
}

\section{To cite this version:}

V. Kochereshko, E. Ivchenko, A.V. Kavokin, P. Kopev, N. Ledentsov. Exciton resonance reflectivity study of quantum well wires. Journal de Physique IV Proceedings, 1993, 03 (C5), pp.C5-363-C5-366. 10.1051/jp4:1993576 . jpa-00251663

\section{HAL Id: jpa-00251663 https://hal.science/jpa-00251663}

Submitted on 1 Jan 1993

HAL is a multi-disciplinary open access archive for the deposit and dissemination of scientific research documents, whether they are published or not. The documents may come from teaching and research institutions in France or abroad, or from public or private research centers.
L'archive ouverte pluridisciplinaire HAL, est destinée au dépôt et à la diffusion de documents scientifiques de niveau recherche, publiés ou non, émanant des établissements d'enseignement et de recherche français ou étrangers, des laboratoires publics ou privés. 


\title{
Exciton resonance reflectivity study of quantum well wires
}

\author{
V.P. KOCHERESHKO, E.L. IVCHENKO, A.V KAVOKIN, P.S. KOPEV and N.N. LEDENTSOV \\ A.F. Ioffe Physico-Technical Institute, 194021 St. Petersburg, Russia
}

\begin{abstract}
We report on the first observation of the exciton resonant reflection from a directly grown array of isolated quantum-well-wires. Structures contaned $30 \mathrm{GaAs} / \mathrm{AlAs}$ double layers were grown on (311) GaAs substrate by conventional elemental source MBE. GaAs wire-like clasters with an orientation along [233] and size of 32/10.2 $\AA$ were introduced inside one of the central AlAs layers. The expected distance between nearest clusters is about $160 \AA$. In spite of much smaller effective volume occupied by excitons in these clusters, as compared to that in a single quantum well, the quantum-well-wire structures demonstrate much lager resonance amplitude of the reflection spectra. We extracted excitonic parameters from these spectra and found that the exciton oscillator strength in the quantum-well-wires is more than 10 time higer than that in the enviroment superlattice. A $30 \%$ anisotropy of the exciton oscillator strength in the quantum-well-wire have been observed. The theory of non-local dielectric responce is extended to calculate reflection spectra from the structures with a grating of quantum-wellwires. A functional relationship between the reflection coefficient and the envelope wave function of an exciton in the structure has been established.
\end{abstract}

Quantum wire and quantum dot structures attract growing interest in the recent years [1]. The interest to such structures is caused by potential technical applications and conditions for novel physical phenomena arised due to the reduction of dimentionality. Such novel physical phenomena are connected first of all with the modification of interaction between quasi-particles (electron-electron, electron-hole, electron-phonon, e.t.c.) in low-dimensional systems. In the present paper we present the results of theoretical and experimental investigation of excitonic states in the system of quantum well wires.

The samples contained $30 \mathrm{GaAs} / \mathrm{AlAs}$ double layers grown on (311)A GaAs substrate by conventional-source MBE (Fig.1). In this case, an interface is formed by a periodic array of upward and downward steps oriented along [233] with lateral periodicity of $32 \AA$ and step height of $10.2 \AA$ [2]. The average thicknesses of GaAs and AlAs layers were $35 \AA$ and $30 \AA$, respectively. GaAs wire-like clasters were introduced incide one of the central AlAs layers in the way described in [3,4]. According to [3] the clusters (covering $-20 \%$ of the layer surface) are responsible for the formation of wire-like quasy-one 


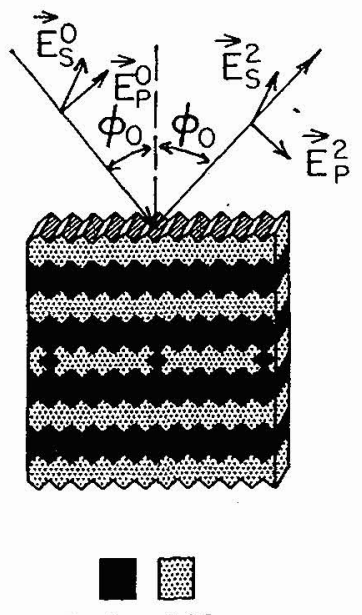

GaAs AlAs

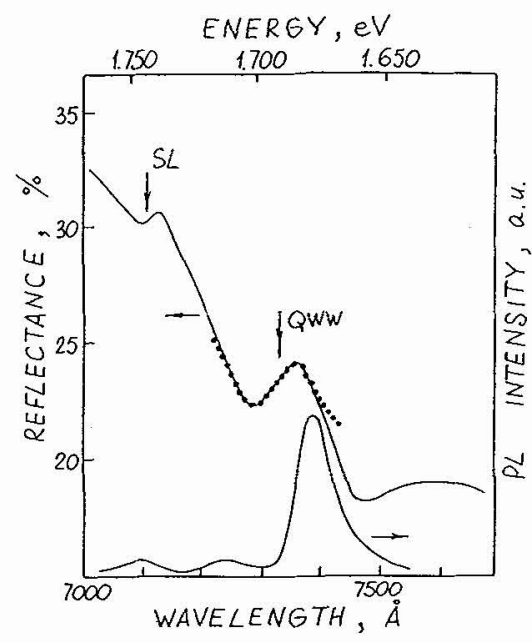

Fig. 1 Schematic representation of light reflection geometry and (311)A corrugated superlattice with wire-like clusters introduced inside one of AlAs layers $x\|[01 \overline{1}], y\|[\overline{2} 33]$.

Fig. 2 Normal reflection and photoluminescence spectra taken from a GaAs/AlAs multy-layered structures with a grating of wire-like clusters. Dotted line shows the reflection spectrum in the vicinity of $\omega_{0}^{Q W W}$ with fitting parameters: $\hbar \omega_{0}^{Q W W}=1.690 \mathrm{eV},(2 \gamma)^{-1}=3 \mathrm{ps}, \hbar \Gamma=6.6 \mathrm{meV}$.

dimensional (quasy-1D) free-carrier states. The expected distance between nearest clusters $d_{x}$ is $160 \AA$ and the interaction of quasi-1D excitonic states seems to be negligible.

Fig. 2 shows the reflection spectrum taken at normal incidence in the $E \| y$ polarization ( $E$-electric vector of reflected light, $y \|[\overline{2} 33]$ ) together with the spectrum of photoluminescence excited by the $6328 \AA$ line of a He-Ne laser. The arrows indicate the resonance frequences of ground-state excitons in the corrugated superlattice $\left(\omega_{o}^{S L}\right)$ and of quasi-1D excitons attached to wire-like clusters $\left(\omega_{o}^{Q W W}\right)$. We note the large intensity of photoluminescence signal from QWW clasters in comparison with superlattice.

The reflectivity spectrum was calculated in the framework of the theory of non-local dielectric response. The dotted curve is calculated using the expression for the interference reflectivity (see [5]).

$$
R=r_{01}^{2}+2 t_{01} t_{10} r_{01} \operatorname{Re}\left(e^{i \phi} r\right)
$$

where $r_{01}, t_{01}, t_{10}$ are the amplitude reflection and transmission coefficients on the external boundary, $\phi$ is the phase shift of the light wave after its direct and backward passage from the external boundary to the wire-like clusters, the resonance reflection coefficient $r$ is given by following equation:

$$
r_{s, p}=\frac{i \gamma_{s, p}}{\omega_{0}-\omega-i \Gamma}
$$


here $\gamma_{s}=\gamma / \cos \phi, \gamma_{p}=\gamma_{s} \cos 2 \phi$ for $\mathrm{-} \mathrm{and} \mathrm{p}$ - polarization respectively,

$$
\gamma^{Q W W}=\left(Q / d_{x}\right)\left[\int \Phi_{Q W W}(\rho) d \rho\right]^{2}
$$

$Q=k \omega_{L T} \pi a^{2} / 2$ and, for simplicity, we assumed that $k a, k d_{x, y} \ll 1$ where $a$ is the QWW width along $z$. In this case the light diffraction by the array of QWWs is absent and the exciton oscillator strength in reflection $\gamma_{\eta}$, coincide with $\left(2 \tau_{\eta}\right)^{-1}, \tau_{\eta}$ being the radiative lifetime of $\eta$-polarized excitons. Note that $\gamma$ differs from radiative dumping of an exciton in a single QWW. In particular $\gamma^{Q W W}=\left(2 / k d_{x}\right) \gamma_{y}^{S Q W W}, \gamma_{y}^{S Q W W}=2 \gamma_{x}^{S Q W W}$

Fig.3 demonstrates the lineshape inversion in the reflection spectrum from the wire-like clusters at the Bruester angle $\phi_{B r}$. The inversion takes place because the reflection coefficient on the external boundary, $r_{01}$, changes its sign at $\phi=\phi_{B r}$.

We would like to note a very large amplitude of resonance lineshape of exciton in quantum well wire in comparison with corrugative superlattice. The large value of the modulation indicates the large increasing of the oscillator strength of exciton in $1 \mathrm{D}$ structure.

The obtained value of exciton radiative lifetime, $\tau_{Q W W}=\left(2 \gamma^{Q W W}\right)=3$ ps, appears to be very short as compared with that for excitons in SQWs: $\tau_{S Q W}=11$ ps for a GaAs/AlAs single quantum well of thickness $a=30 \AA, \tau_{S Q W}=10$ ps if $a=50 \AA$ or $100 \AA$. This signify much stronger exciton confinement in
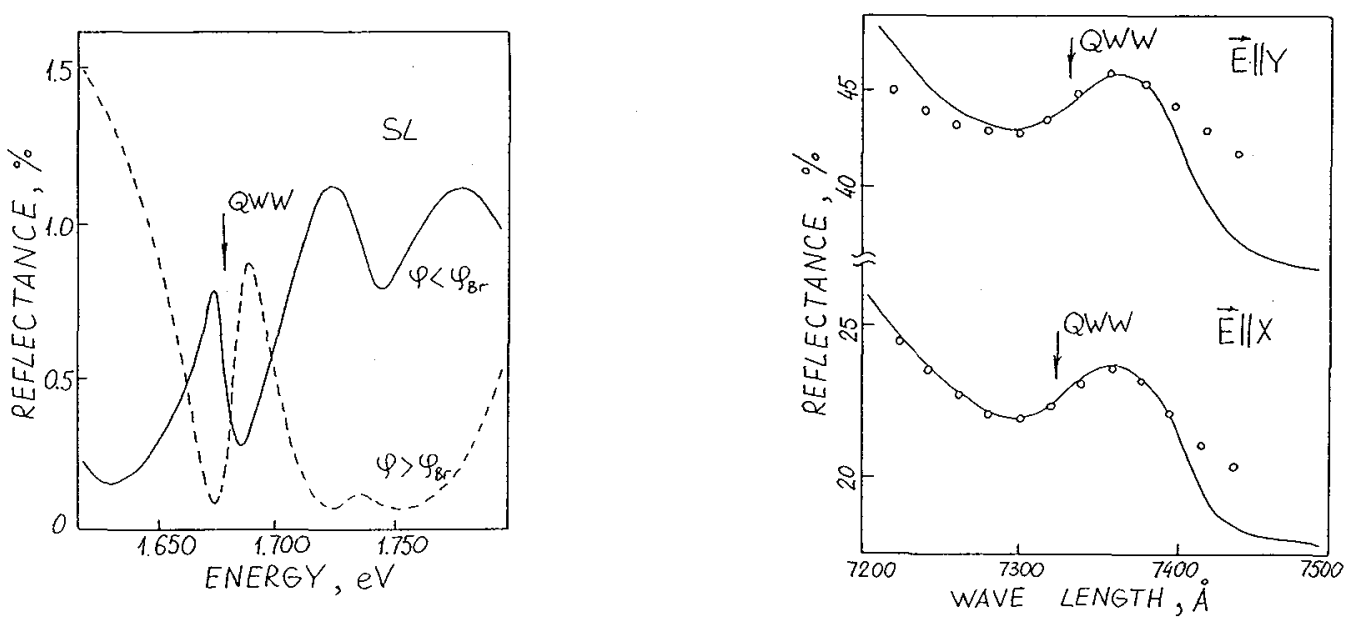

Fig.3 Oblique reflection spectra measured in the $p$-polarization at the incidence angle close to the Bruester angle.

Fig.4 Anisotropy of reflection spectra, of QWW clasters. y-direction along wire-like clasters, $\mathrm{x}$ perpendicular direction. 
the structure under investigation than in a quantum well and evidences the existence of quasy-1D exciton states in our structure. Moreover, the experimental value of $\tau_{Q W W}$ is in reasonable agreement with the provided theoretical estimations.

The characteristic peculiarity of these structures is the large value of anisotropy of reflectivity coefficient. That is, the reflectivity coefficient for the light polarization parallel to the wire direction ( $E\|[\overline{2} 33]\| y$ ) is larger than for perpendicular polarization (Fig.4). This anisotropy of reflectance displays not only the anisotropy of QWW exciton resonance contribution to reflectance but the anisotropy of background reflectiviry also. We suppose that the anisotropy of background reflectivity display the optical anisotropy of the bulk cubic crystals in the nonsymmetrical orientations [311] and can be discribed by the mixing of the heavy- and light- hole states.

In addition to the anisotropy of background reflectivity we have found the remarkable anisotropy of the exciton resonance contribution to reflectance. By fitting the calculated and experimental spectra we have found the difference in the exciton oscillator strength for light polarized with $E \| x$ and $E \| y: \gamma_{y}^{Q W W} / \gamma_{x}^{Q W W}=1.5$. This walue is in agreement with the theoretical prediction for the $a / b=0.6$. ( $a$ - is a width of QWW along $z$ direction, $b$-is a width of QWW along $x$ direction (see Fig.1)).

In conclusion, the resonant reflectivity from the directly grown array of isolated QWWs have been observed. In spite of the much smaller effective volume occupaied by excitons as compared to that in a SQW, the QWW structure demonstrates essentialy higher exciton oscillator strength. The 30\% anisotropy of exciton oscillator strength being found for QWW demonstrates quasi-1D character of these excitonic states.

Acknowledgement: This work has been supported in part by the Soros Foundation Grant.

\section{References}

[1] Heitmann D., Demel T., Grambow P., Kohl M., Ploog K. Proc.20 ICPS, Thessaloniki, (1990), p.13.

[2] Notzel R., Ledentsov N.N, Daweritz L.A., Hohenstein M., Ploog K. Phys.Rev.Lett. 68, (1991), 3812.

[3] Ledentsov N.N., Bert N.A., Egorov A.Yu., Ivanov S.A., Kop'ev P.S., Meltser B.Ya., Ruvimov

S.A.Tabatadze I.G., Ustinov V.M., Zhukov A.E., J.Crystal Gowth 129, (1992), 456.

[4] Ledentsov N.N., International School on Optical Properties of Semiconductors, Erice, Italy, (1992).

[5] Ivchenko E.L., Kochereshko V.P., Uraltsev I.N. in: Semiconductor and Insulators: Optical and Spectroscopic Research. ed. by Yu.I.Koptev, Nova Science Publishers (1992). 